\title{
SURFACE MICROMACHINE MICROFLUIDICS: DESIGN, FABRICATION, PACKAGING, AND CHARACTERIZATION
}

\author{
Paul Galambos ${ }^{*}$, William P. Eaton, Randy Shul, Christi Gober Willison, Jeffrey J. \\ Sniegowski, Samuel L. Miller \\ and Daniel Guttierez of Sandia National Labs, PO Box 5800, MS 1080, Albuquerque NM \\ 87185-1080 \\ http://www.mdl.sandia.gov/Micromachine, "(505)844-1542, FAX (505)844-2991,
} pcgalam@sandia.gov

\begin{abstract}
The field of microfluidics is undergoing rapid growth in terms of new device and system development. Among the many methods of fabricating microfluidic devices and systems, surface micromachining is relatively underrepresented due to; difficulties in the introduction of fluids into the very small channels produced, packaging problems, and difficulties in device and system characterization. The potential advantages of using surface micromachining include: compatibility with the existing integrated circuit tool set, integration of electronic sensing and actuation with microfluidics, and fluid volume minimization. In order to explore these potential advantages we have developed first generation surface micromachined microfluidic devices (channels) using an adapted pressure sensor fabrication process to produce silicon nitride channels, and the SUMMiT process to produce polysilicon channels. The channels were characterized by leak testing and flow rate vs. pressure measurements. The fabrication processes used and results of these tests are reported in this paper.
\end{abstract}

\section{INTRODUCTION}

The field of microfluidics is experiencing rapid growth in terms of both basic research and device development. A variety of microfluidic devices are being developed for applications ranging from HTAS (Micro-Total (chemical) Analysis Systems, Harrison 1998), to ink jet printing (Kamisuki 1998). These devices are fabricated using a wide range of technologies including: bulk micromachining (e.g. $\mathrm{KOH}$ etch), high aspect ratio micromachining (e.g. DRIE or LIGA), laser machining, very small scale conventional machining (drilling and milling), capillary tubing assembly and various unconventional techniques (Whitesides 1997). Conspicuously absent from this list is surface micromaching.
Consider as a representative sample the microfluidic papers presented at the ASME winter annual meeting last October (Forster et. al. 1998). Of the 39 papers dealing with experimental microfluidic devices only 3 utilized surface micromachining. Of these 3 papers one (Tseng 1998) was a hybrid design utilizing both bulk and surface micromachining with the flow channels fabricated utilizing bulk micromachining. The 2nd paper utilized surface micromachining to produce valves that controlled flow in much larger passages (Wroblewski 1998). Only the $3^{\text {rd }}$ paper (Rasmussen 1998) described flow channels fabricated using surface micromachining. Therefore only $1 / 39$ or $2.6 \%$ of the microfluidic papers presented (Forster et. al. 1998) dealt with a microfluidic system in which the primary flow channels were fabricated using surface micromachining.

Despite this lack of utilization, surface micromachining has significant potential advantages over other fabrication techniques in some microfluidic applications. One advantage is volume minimization. A typical bulk micromachined microfluidic device has a channel depth on the order of $100 \mu \mathrm{m}$ and a volume on the order of $100 \mathrm{nl}$ (assuming an approximately $500 \mu \mathrm{m}$ wide by $1 \mathrm{~mm}$ long channel). A typical surface micromachined channel is only 2 to $5 \mu \mathrm{m}$ deep, $200 \mu \mathrm{m}$ wide and $1 \mathrm{~mm}$ long for a volume on the order of $1 \mathrm{nl}$ ( 2 orders of magnitude smaller). In applications where volume minimization is important (such as $\mu$ TAS) this 2 order of magnitude difference could be significant in reducing mixing times and reagent requirements.

Surface micromachined MEMS devices have been developed with sophisticated electrostatic actuation systems for use in a wide variety of devices ranging from microengines (Garcia 1995) to accelerometers and gyros (Allen 1998). CMOS electronics and MEMS have been integrated on a single silicon 


\section{DISCLAIMER}

This report was prepared as an account of work sponsored by an agency of the United States Government. Neither the United States Government nor any agency thereof, nor any of their employees, make any warranty, express or implied, or assumes any legal liability or responsibility for the accuracy, completeness, or usefulness of any information, apparatus, product, or process disclosed, or represents that its use would not infringe privately owned rights. Reference herein to any specific commercial product, process, or service by trade name, trademark, manufacturer, or otherwise does not necessarily constitute or imply its endorsement, recommendation, or favoring by the United States Government or any agency thereof. The views and opinions of authors expressed herein do not necessarily state or reflect those of the United States Government or any agency thereof. 


\section{DISCLAIMER}

Portions of this document may be illegible in electronic image products. Images are produced from the best available original document. 
substrate in an IMEMS (integrated MEMS) process (Smith 1995). In a similar manner surface micromachined microfluidics can potentially be integrated with electronics to produce electromicrofluidic devices. The additional integration of microfluidics onto a single silicon substrate containing MEMS and/or electronics can lead to a new class of devices, electro-microfluidic MEMS systems on a chip. This class of devices has the potential to accomplish a wide variety of functions (e.g. power generation, $\mu$ TAS, hydraulic actuation and control) in a very compact package.

Perhaps the most important reason to develop surface micromachined microfluidics has to do with the tool set used in their manufacture (Smith 1998). This tool set is highly developed for use in IC manufacturing. For this reason it should be relatively easy to go from prototype to mass production with a surface micromachined microfluidic device. In principle such devices can be manufactured as cheaply as electronic integrated circuits.

Before any of these advantages can be realized the packaging challenge must be met. Due to the very small channels produced by surface micromachining $(<5 \mu \mathrm{m})$, it is very difficult to get fluid into the channels and seal the interface. There is presently no standard method for packaging surface microfluidic devices.

In addition, the performance of surface micromachined microfluidic devices has typically not been completely characterized. In order to use these devices as part of a larger system on a chip or to interface microfluidic devices on a chip with devices external to the chip, the performance characteristics of the microfluidic devices must be quantified. The simplest microfluidic device is a microfluidic channel. Therefore we will focus on the design, fabrication and characterization of very simple microfluidic devices, surface micromachined flow channels. For such simple devices performance is characterized primarily by flow resistance. In this paper, we will attempt to characterize surface micromachined microfluidic channels in terms of flow resistance.

\section{DESIGN AND FABRICATION}

For surface micromachining in general, surface micromachined MEMS, and surface micromachined microfluidics, device design and fabrication are intimately linked. Device design is constrained by the fabrication process used. In this research two processes were applied to produce two different types of microfluidic channels. Type I channels were fabricated in silicon nitride and were produced using a process developed to manufacture pressure transducers (Eaton 1997). Type II channels were fabricated in polysilicon using SUMMiT (www.mdl.sandia.gov/Micromachine). The process flow for type I channels is shown in Fig. 1 (Eaton 1997).

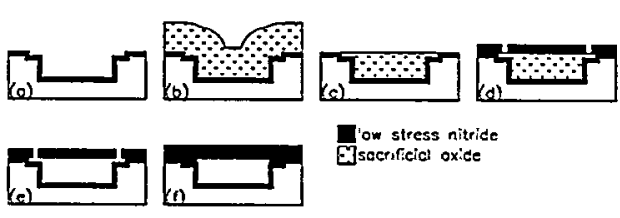

Figure 1. Process Flow for Type I Channels (Silicon Nitride). The fabrication process begins with a trench etched in a silicon wafer and lined with low stress silicon nitride (nitride) (a). The trench is filled with silicon dioxide (oxide) (b). The oxide is polished so that it is even with the top of the nitride using CMP (Chemical-Mechanical Polishing) (c). A thicker layer of nitride is deposited and patterned with etch release holes (d). The sacrificial oxide is removed in an HF (Hydrofluoric acid) release etch (e). The etch release holes are filled during the final nitride deposition ( $f$ ).

The process begins with a $2 \mu \mathrm{m}$ trench etch in a silicon wafer. The trenches are defined using a patterned TEOS (thermal oxide) hard mask. After etching the trenches the TEOS mask is stripped in HF (Hydrofluoric acid) and a $0.3 \mu \mathrm{m}$ thick layer of low stress silicon nitride (nitride) is deposited using Low Pressure Chemical Vapor Deposition (LPCVD). After a sacrificial oxide refill the trenches are chemicalmechanically polished (CMP) flat. A $0.8 \mu \mathrm{m}$ thick low stress nitride membrane (channel cover) is then deposited over the oxide. The membrane is patterned with etch release holes using another TEOS hard mask, and the release holes are etched using a dry etch. After stripping the hard mask, the structures are released in a 1:1 $\mathrm{HF}: \mathrm{HCl}$ (Hydrofluoric:Hydrochloric) acid bath. The solution is highly selective - etching the oxide while leaving the nitride alone. Finally the etch release holes are sealed using another LPCVD nitride deposition. The resulting channels are approximately $2 \mu \mathrm{m}$ deep. The channels are $200 \mu \mathrm{m}$ wide at the inlet and outlet and neck down to various widths in between. Fig. 2 is a photograph of a channel and Fig. 3 is a SEM (scanning electron micrograph) image of a channel cross-section.

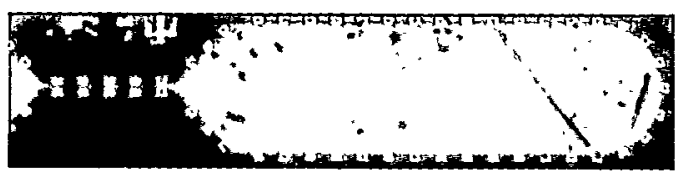

Figure 2. Photograph of Type I channel. The etch release holes are spaced around the channel edge. The channel necks down to $10 \mu \mathrm{m}$ in width at the left and is $200 \mu \mathrm{m}$ wide where the Bosch etch hole intersects the channel at the right. 


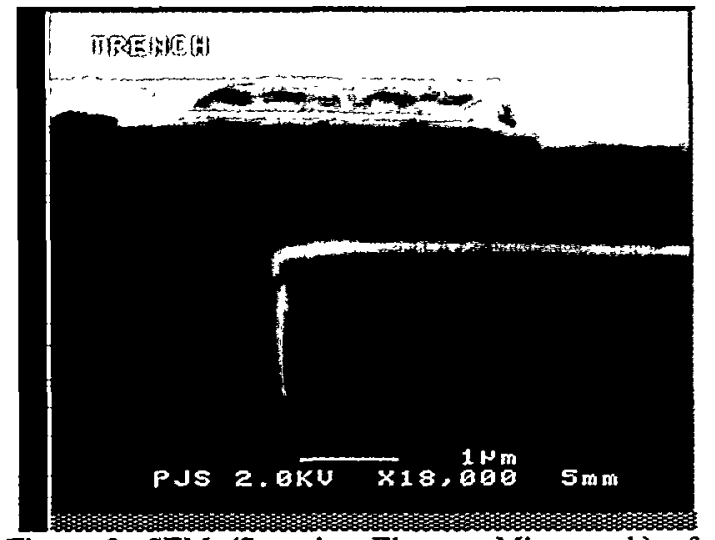

Figure 3. SEM (Scanning Electron Micrograph) of type I channel. The vertical walled channel is slightly less than $2 \mu \mathrm{m}$ deep, and the bottom and sides are lined with $0.3 \mu \mathrm{m}$ thick nitride. The top is a $1 \mu \mathrm{m}$ thick nitride membrane.

In order to introduce fluid at the channel inlet and outlet, via were etched through the wafers from the back using a Bosch process (Bosch 1996). The Bosch process is a Deep Reactive Ion Etch (DRIE) that relies on an iterative deposition/etch cycle in which a polymer etch inhibitor is conformally deposited over the wafer during deposition cycles. The polymer is preferential removed from the bottom of the via due to acceleration of ions perpendicular to the surface of the wafer. Therefore the sides of the via are protected and a vertical walled via results. The Bosch etch via extended all the way through the wafer and were stopped by the nitride layer on the bottom of the type I channels. These via were $200 \mu \mathrm{m}$ in diameter. Finally the nitride membrane on the bottom of the channel were removed using either a longer exposure to the Bosch process or a Plasma-Therm parallel plate RIE system.

The process flow for type II channels is shown in Fig. 4. A two-level channel is shown. The first level is in the first structural layer of polysilicon (POLY1) and the second level (top channel cover) is in the $3^{\text {rd }}$ structural layer of polysilicon (POLY3). The $2^{\text {nd }}$ layer of structural polysilicon (POLY2) is used to fabricate a channel for acid flow during release. Sacrificial oxide layers define the depth of the channels (SACOX1 and SACOX3). Another oxide layer (SACOX2) defines the acid flow channel. During the release process the thin layer of SACOX2 allows acid to flow inside the channels and remove the sacrificial oxide (Silicon Dioxide). Not shown in Fig. 4 is a passage that is defined in POLY2 that allows the acid to attack the oxide between POLY1 and POLY3. Details of the SUMMiT process can be found on the web (www.mdl.sandia.gov/Micromachine).

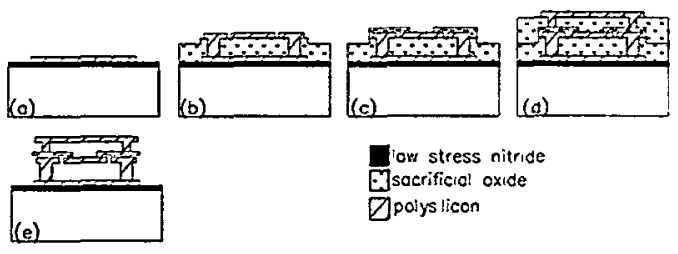

Figure 4. Process Flow for Type II channels (polysilicon). The process begins with deposition of low stress nitride and the bottom layer of polysilicon (POLY0) (a). The first layer of sacrificial oxide (SACOX1) is then deposited, patterened and etched to produce an anchor for the second layer of polysilicon (POLY1) (b). POLY1 is then deposited patterned and etched to produce a channel cover with etch release holes (b). A second layer of oxide (SACOX2) is deposited, patterned and etched (c). A $3^{\text {rd }}$ polysilicon layer (POLY2) is deposited over SACOX2 and anchored to POLY1 (c). Another layer of oxide (SACOX3) is deposited and etched for anchoring the $4^{\text {th }}$ level of poly (POLY3) (d). POLY3 is deposited to form the top cover of the channel (d). Finally the structure is released by etching away the sacrificial oxide in an HF:HCI bath (e). The channels will be sealed with a sputter deposition of silicon dioxide.

Several different versions of the type II channels in addition to the two level channel shown in Fig. 4 were designed. All of the other designs consisted of one level channels: one design with a POLY1 cover, one with a POLY2 cover, one with a POLY1/2 laminated cover and one with a POLY 3 cover. This will result in channel depths of approximately $1.5,2.0$, and 4.5 microns for the various channels.

Type II channels were also Bosch etched from the back side at the channel inlet and outlet to allow fluid to enter and exit. In the SUMMiT process the bottom layer of nitride was removed at the channel inlet and exit using a cut in the nitride layer. Therefore the Bosch etch is stopped on the first layer of oxide (SACOX1) rather than the nitride. During etch release the channel is hollowed out and the Bosch etch hole from the back is uncovered allowing fluid to enter the channels.

\section{PACKAGING}

Packaging of microfluidic systems is particularly challenging because of the very small size of the fluid channels and sealing requirements. In addition, for these devices we wanted to make the package separate from the surface micromachined microfluidic parts in order to allow different 
microfluidic devices to be tested with the same packaging setup. Therefore this packaging arrangement is a test fixture for microfluidic devices rather than a production package for inclusion in a larger system.

Both type I and type II channels were fabricated on approximately $5 \mathrm{~mm}$ by $5 \mathrm{~mm}$ silicon die. They each contained approximately 16 inlet and outlet connections corresponding to approximately 8 channels per die. Each die contains multiple fluid connections, therefore a flow manifold rather than individual microfluidic connectors was utilized. The flow manifold was fabricated from aluminum. The packaging arrangment utilizing the flow manifold is shown in Fig. 5.

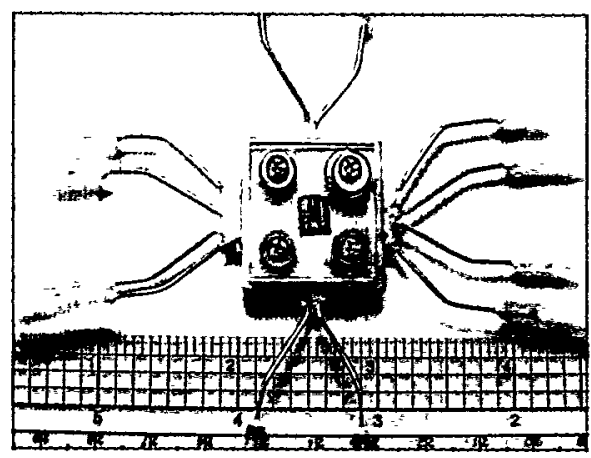

Figure 5. Packaging Arrangement. The $5 \mathrm{~mm}$ by 5 $\mathrm{mm}$ microfluidic die is shown in the center of the photograph mounted onto the 1 in by 1 in aluminum manifold. The die is held in place by means of a clamping plexiglass plate. The syringe needles allow connection of external fluid lines.

The aluminum flow manifold contains very small drilled holes in the same pattern as the Bosch etch holes in the silicon wafer. These holes intersect larger perpendicular holes drilled from the sides of the manifold. These larger holes are sized to provide a tight fit for $\mathbf{2 0}$ gage syringe needles which are epoxied into the holes for sealing. The syringe needle fittings then provide a relatively convenient method for attaching external fluid lines to the manifold.

The holes in the manifold that align with the Bosch holes are $200 \mu \mathrm{m}$ in diameter and approximately $1 \mathrm{~mm}$ apart. A counterbore in the aluminum manifold allows $200 \mu \mathrm{m}$ inner diameter O-rings (Apple Rubber Products, Lancaster NY) to be placed around these manifold holes. The silicon die is then aligned with the O-ring surrounded holes and clamped down using a plexiglass plate with clamping screws into the manifold. The O-rings compress - sealing the joint between the manifold and the die around each separate fluidic connection. This arrangement allows separate fluids to be plumbed to each microfluidic device on the chip. The resulting sandwich of aluminum manifold, silicon die, and plexiglass cover is leak tight.

The alignment between the manifold and the silicon die is accomplished optically using a low power microscope. The die is placed on top of the manifold with the O-rings in place. A light shines from below through alignment holes in the manifold. These alignment holes line up with corresponding alignment holes in the die allowing precise optical alignment $(+/-$ $10 \mu \mathrm{m}$ ). The $3.175 \mathrm{~mm}$ thick plexiglass plate is then placed over the die and tightened to the manifold using 4 screws to complete the assembly.

\section{DEVICE CHARACTERIZATION}

As microfluidics moves out of the laboratory and into industrial, commercial and military application device reliability and performance become more and more important. Almost all microfluidic devices at the simplest level are concerned with flow through very small flow passages. Therefore one reasonable way of characterizing microfluidic devices is in terms of their flow rate and the pressure required to achieve that flow rate. For electrically powered flows it makes more sense to measure flow rate as a function of voltage, current or power. However in either case, the flow resistance of the channels characterizes the severity of viscous effects.

In addition microfluidic systems can be characterized in terms of how leak tight they are. This is particularly the case for gas flow systems. In liquid systems surface tension acts to prevent leaks, and at microfluidic scales surface tension is a significant force. On the other hand the pressures developed even at very low flow rates are significant in microfluidic systems. Therefore microfluidic systems must be leak tight to very high pressures.

For any characterization the entire package must be considered. When checking for leaks, for instance, the characterization is not so much of the surface micromachined microchannels but of the die/packaging assembly. In addition the characteristics of the measurement system must be considered when evaluating the microfluidic system. For instance when using a syringe pump to develop a controlled flow rate in a microfluidic channel the large volume of the syringe relative to the microfluidic channel must be considered.

\section{Package Sealing}

Package sealing was characterized using two techniques; a helium leak test apparatus, and a gas tight syringe pump. The vacuum test apparatus involves pulling a vacuum inside the microfluidic channels and testing for leakage of helium from outside the channels to inside. The gas tight syringe 
tests involved pressurizing the channels using the syringe pump and checking measured pressure vs. volume characteristics vs. those predicted using the ideal gas law for a sealed container. Deviations from ideal gas low predictions can be related to a leak rate.

In terms of liquid flow, leak rates above the evaporation rate can be determined from observation. Pressures as high as $50 \mathrm{Atm}$ were measured with no observable liquid leak. The evaporation rate was measured as approximately $10 \mathrm{nl} / \mathrm{s}$ by weighing a volume of water with a sensitive scale as it evaporated from an approximately $1.5 \mathrm{~cm}$ diameter graduated cylinder. The surface area of the water exposed to evaporation was approximately $1.77 \times 10^{-4} \mathrm{~m}^{2}$ during the evaporation measurement. The evaporation rate per unit area was therefore approximately $56 \mu l / \mathrm{m}^{2} s$. The area for evaporation between the 0 -ring and the manifold was estimated at approximately $6.3 \times 10^{-10}$ $\mathrm{m}^{2}$ (assuming a $1 \mu \mathrm{m}$ gap around the O-ring outer diameter). Therefore the evaporation rate for the manifold assembly is estimated as $35 \mathrm{fl} / \mathrm{s}$. The reason the evaporation rate is so low is that evaporation can only occur in the very small gap between the O-ring and the manifold or silicon wafer interfaces.

Helium Leak Tests. The manifold assembly was leak tested using a vacuum leak test machine (Varian Vacuum Products, Lexington MA). A sealed stainless steel tubing line connected the microfluidic assembly to the leak testing machine. The connecting line was verified as leak tight using the leak testing machine. The line was then attached to one flow channel input line and the connection was sealed. The downstream exit from the channel was sealed at the manifold exit of the channel.

The first step of the leak check process was to suck down the sealed volume, which includes the microfluidic channel, its inlet and outlet manifold passages, and the connecting tubing. This volume was evacuated to a pressure of $<1 \mathrm{mtorr}$, which is approximately $0.001 \%$ of an atmosphere, using a vacuum pump built into the vacuum tester. Therefore there is a pressure difference of approximately $1 \mathrm{Atm}$ between the inside and outside of the sealed volume. This level of pressure difference is required for the vacuum tester to operate.

A separate helium line is then used to introduce helium around the device. The exit of the helium line was a small needle that was used to place helium precisely at all the joints of the assembly. A mass spectrometer then measured the amount of helium that leaked through the assembly into the connecting tubing to reach the mass spectrometer. Five measurements were taken on three different manifold assemblies. A leak rate of:

$$
1.6 \pm 4 \times 10^{-11} \mathrm{l} / \mathrm{s} \mathrm{Atm}
$$

of helium was measured. This leak rate applies for conditions of approximately $1 \mathrm{Atm}$. of Vacuum in the channel.

Syringe Pump Leak Tests. Leak tests for positive pressure difference (pressure inside the channel higher than atmospheric) were conducted using a gas tight $50 \mathrm{ml}$ syringe (Hamilton Syringe, Reno NV) and a syringe pump (Harvard Apparatus, Cambridge MA) to pressurize air contained in the combined syringe, manifold, channel and connecting tubing volume. The manifold channel exiting the microchannel was sealed providing a closed volume.

The nitride membrane channel covers deflect under the positive pressure (Fig. 6). From the interference fringe pattern shown ( 3 fringes) a maximum membrane deflection of approximately $0.8 \mu \mathrm{m}$ was calculated.

$$
d=3 \times \frac{\lambda_{\text {avg }}}{2}=825 \mathrm{~nm}
$$

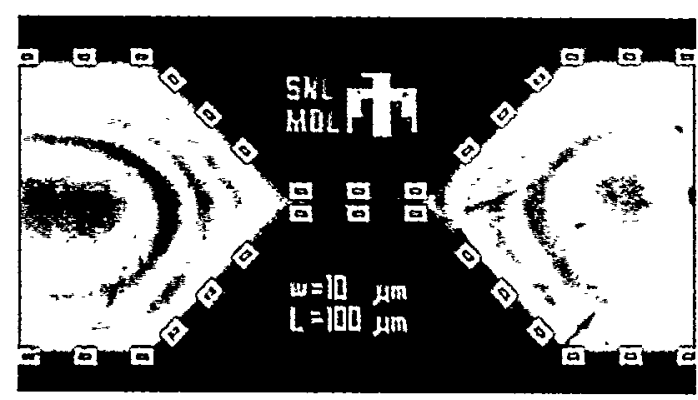

Figure 6. Leak Test Membrane Deflection. Type I channel pressurized to approximately $3.5 \mathrm{Atm}$. Light passing through the channel cover reflects off the bottom of the channel and cancels or enhances light reflected off the channel cover to produce the fringe pattern.

Pressure measurements just upstream of the microchannel manifold were used to calculate the excess mass of air contained in the volume. The excess mass of air is the mass of air in addition to that which would be contained in the volume at atmospheric pressure. Gauge pressure was measured at an in-line pressure measurement $T$ between the syringe pump and the microfluidic manifold. One leg of the $\mathrm{T}$ connected to a very small pressure transducer (Entran Devices Inc., Fairfield NJ) and the other two legs provided the inlet and outlet for the feed through.

As air leaked from the volume the gauge pressure dropped (Fig. 7). Two different microfluidic manifold assemblies were tested. The 4/10/1999 data was taken on a manifold assembly that contained a gasket spacer between the plexiglass cover and the manifold. The 
$3 / 12 / 1999$ data manifold assembly did not utilize the spacer. The gasket spacer provided more even clamping of the plexiglass cover plate. The $4 / 10$ data did not show the nitride membrane deflection that the $3 / 12$ data did (Fig. 6), due to this more even clamping.

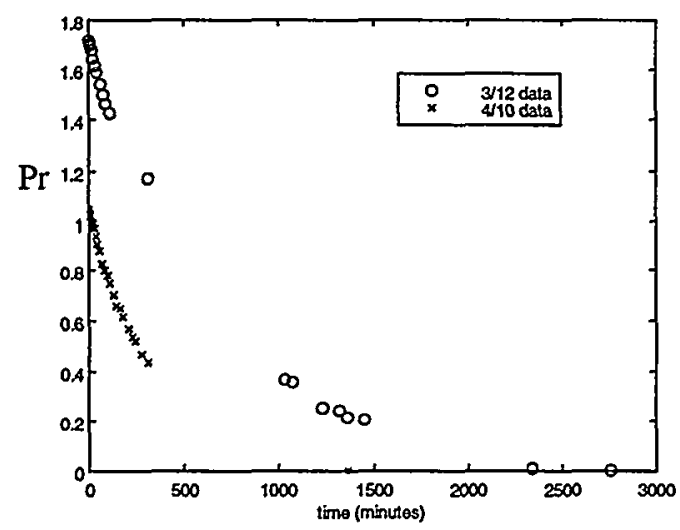

Figure 7. Leak Test Data. Pressure ratio, (P-Patm)/ Patm, decays as air leaks out of two different microfluidic manifold assemblies.

The exponential decay in pressure was related to the leakage by fitting the curve of mass contained in the volume vs. time. Using the ideal gas law:

$$
\begin{gathered}
m_{\text {excess }}=\frac{P \cdot V}{R_{a i r} T}, R_{a i r}=286 \frac{J}{k g K}, T=295.22 \mathrm{~K} \\
V=28 \mathrm{ml} \quad 3 / 12 \text { data }, V=25.5 \mathrm{ml} \quad 4 / 10 \text { data }(4) \\
m_{\text {excess }}=3.316 \times 10^{-10} \times P \quad 3 / 12 \text { data } \\
m_{\text {excess }}=3.02 \times 10^{-10} \times P \quad 4 / 10 \text { data }
\end{gathered}
$$

The natural $\log$ of this calculated excess mass is plotted vs. time in Fig. 8. The inverse of the slope of the resulting straight lines is the time constant for the exponential decay curves.

$$
m_{\text {excess }}(t)=m_{0} \cdot \exp \left[-\frac{1}{\tau} \cdot t\right]
$$

Differentiate with respect to time to calculate leakage.

$$
\frac{d m_{\text {excess }}(t)}{d t}=-m_{0}\left[\frac{1}{\tau}\right] \exp \left[-\frac{1}{\tau} \cdot t\right]
$$

Leak rates are typically normalized by pressure to get units of $(1 / \mathrm{sec}-\mathrm{atm})$

$$
P(t)=P_{0} \cdot \exp \left[-\frac{1}{\tau} \cdot t\right]
$$

$$
\frac{d m_{\text {excess }}(t)}{d t \cdot P}=-\frac{m_{0} \cdot(1 / \tau)}{P_{0}}
$$

Using this relationship calculate the leak rate (LR),

$$
\begin{aligned}
& L R=0.445 \frac{\mu l}{s \mathrm{Atm}} \quad 3 / 12 \text { data } \\
& L R=0.62 \frac{\mu l}{s \mathrm{Atm}} \quad 4 / 10 \text { data }
\end{aligned}
$$

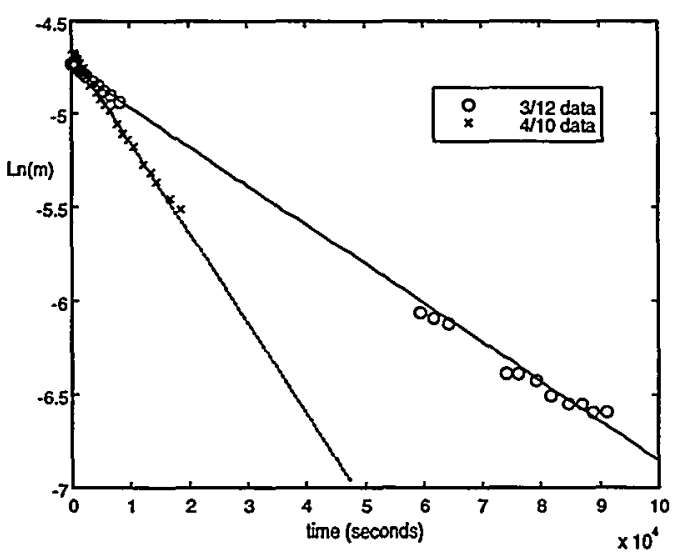

Figure 8. Measured Leak Rates. The slope of the natural $\log$ of the excess mass, $\ln (m)$, decay curve was used to calculate the leak rate.

Air flow in the channel was investigated by opening the downstream manifold passage. The pressure downstream of the flow constriction was atmospheric (see Fig. 9), while the pressure upstream of the flow constriction remained high - approximately $3.5 \mathrm{Atm}$.

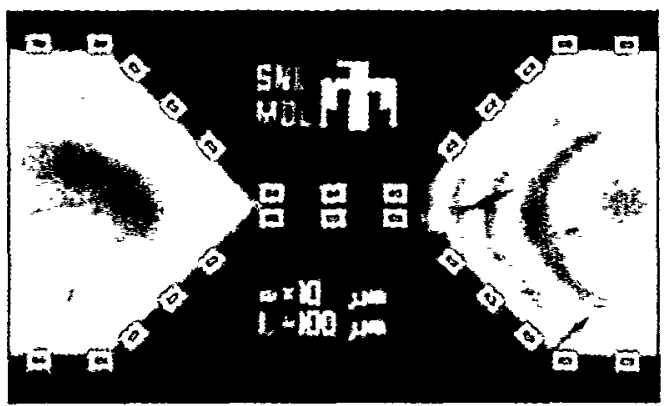

Figure 9. Membrane Deflection - downstream end open. Air flow right to left. The pressure upstream of the narrow section is approximately $3.5 \mathrm{Atm}$. and downstream pressure is approximately $1 \mathrm{Atm}$.

The pressure ratio across the flow constriction was large enough to establish choked flow in the constriction. If we ignore viscous losses:

$$
m_{\text {air }}=\frac{0.0405 \times P_{u p s r e a m}}{\sqrt{T}} \times A_{\text {constriction }}=14 \frac{\mu l}{\mathrm{~s}}
$$


Liquid Flow. Three sets of pressure vs. water flow rate data were measured for three different manifold assemblies. When water was pumped into the first channel/manifold assembly using the syringe pump, an air bubble was trapped in the inlet to the channel (see Fig. 10). In larger flow passages (e.g. a $50 \mu \mathrm{m}$ deep channel or a capillary tube) trapped air bubbles assume a characteristic rounded shape at each end. However in these surface micromachined microfluidic channels the air bubbles assumed an irregular shape. As water flowed through the channel a part of the air bubble broke off and squeezed through the channel. These air bubbles followed regions where the channel was sucked in by pressure (see Fig. 11). The air bubbles moved very rapidly between these sucked in regions. Other bubbles moved through regions that were not sucked in much more slowly.

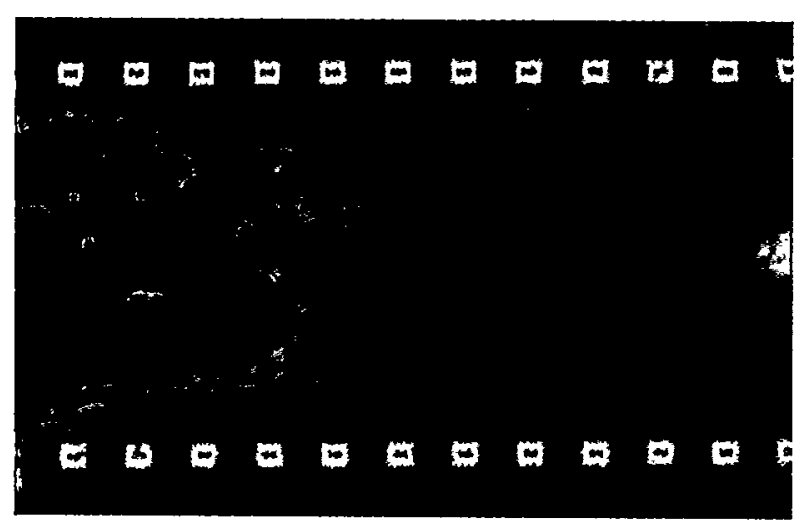

Figure 10. Bubble at Channel Inlet. Water flow from left to right. Channel inlet at far left. The air bubble trapped at the inlet assumes an irregular shape with approximately 3 fringes around it. At the far right a $2^{\text {nd }}$ fringed region is beginning.

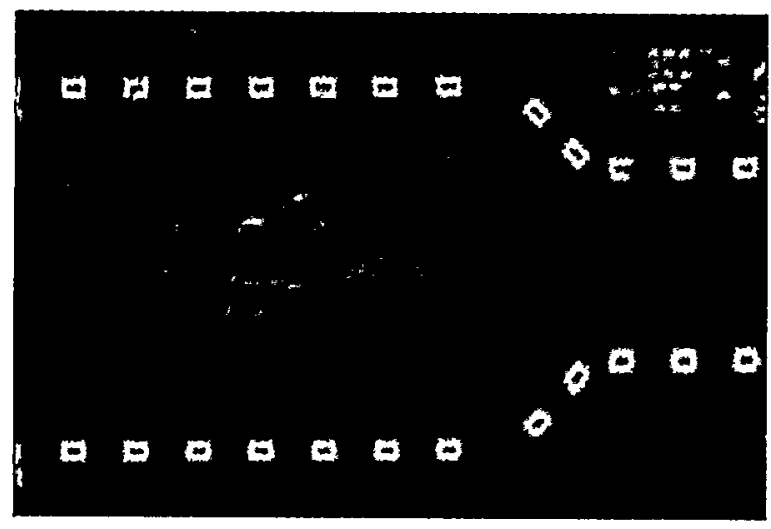

Figure 11 Low Pressure Region Upstream of Flow Constriction. The $2^{\text {nd }}$ fringed region is just upstream of the flow constriction. Large air bubbles move very rapidly between the two low pressure regions and then through the constriction and out the channel exit.
The $2^{\text {nd }}$ and $3^{\text {rd }}$ manifold assemblies tested did not have a bubble trapped at the inlet because they were assembled with water already filling the manifold channels. When the silicon wafer was clamped in position this water was drawn into the channels effectively eliminating any possible bubble formation locations at the manifold/wafer interface. These assemblies did not show bubbles anywhere in the channel after a short flush out period. The difference between the $2^{\text {nd }}$ and $3^{\text {rd }}$ assemblies was the amount of tightening used to attach the clamping plexiglass cover. The $3^{\text {rd }}$ (higher slope case - Fig. 12) was significantly tighter and the channel showed significantly higher flow resistance.

The flow resistance for each assembly was calculated from the slopes of Fig. 12 as:

$$
\begin{aligned}
& 1.08 \times 10^{15} \frac{\mathrm{Ns}}{\mathrm{m}^{5}}, \quad 1 \text { st assembly } \\
& 4.26 \times 10^{15} \frac{\mathrm{Ns}}{\mathrm{m}^{5}}, \quad 2 \text { nd assembly } \\
& 4.15 \times 10^{16} \frac{\mathrm{Ns}}{\mathrm{m}^{5}}, \quad 3 r d \text { assembly }
\end{aligned}
$$

The theoretical flow resistance can be calculated from the Poiseuille slot flow equations (White, 1994).

$$
R_{\text {flow }}=\frac{12 \times \mu}{b^{3}}=\frac{12 \times 10^{-3}}{\left(2 \times 10^{-6}\right)^{3}}=1.5 \times 10^{15} \frac{\mathrm{Ns}}{\mathrm{m}^{5}}
$$

The measured flow resistance for the $1^{\text {st }}$ and $2^{\text {nd }}$ assemblies bracket the theoretical flow resistance. The more tightly clamped $3^{\text {rd }}$ assembly had a significantly higher flow resistance. Fringe patterns were observed in the $3^{\text {rd }}$ assembly nitride membrane indicating that the plexiglass plate was deforming the silicon nitride channel covers, probably causing the higher flow resistance.

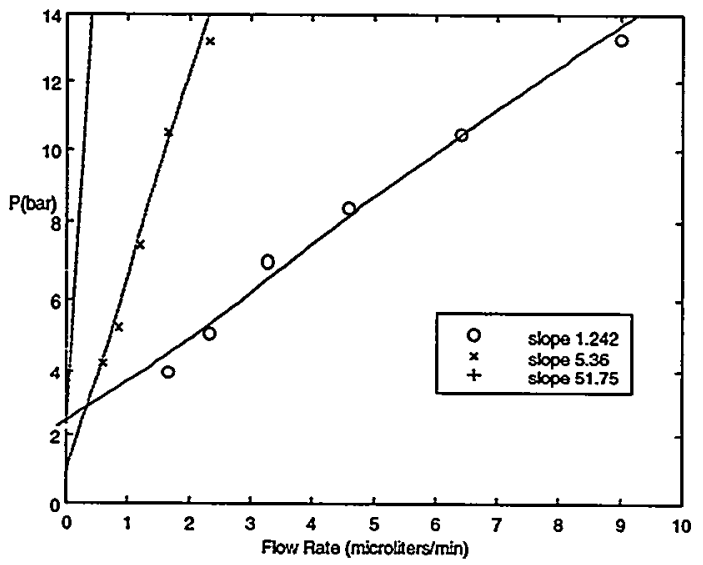

Figure 12. Water Flow Resistance. The slope 1.242 case corresponds to Figs. 10 and 11 . 


\section{ACKNOWLEDGEMENT}

Sandia is a multiprogram laboratory operated by Sandia Corporation, a Lockheed Martin Company, for the United States Department of Energy under Contract DE-AC04-94AL95000.

\section{REFERENCES:}

1. Allen J. J. et al, Integrated Micro-ElectroMechanical Sensor Development for Inertial Applications, PLANS '98, April 1998.

2. Bosch. Patent No. 5501893: Method of Anisotropically Etching Silicon, Robert Bosch Gmblt., Issued 1996.

3. Eaton B., Surface Micromachined Pressure Transducers, PhD Dissertation, University of New Mexico, 1997.

4. Forster et al, Applications of Micro-Fabrication to Fluid Mechanics, Micro-Electro-MechanicalSystems (MEMS) 1998, 1998 ASME International Mechanical Engineering Congress and Exposition, Nov. 1998.

5. Garcia E. J. and J. J. Sniegowski, Sensors and Actuators A 48 (1995), 203-214 (Feb. 1995).

6. Harrison, D. J. et al, Micro-Total Analysis Systems '98, Oct. 1998, Kluwer Academic Publishers.

7. Kamisuki, S. et al, A Low Power Electrostatically Driven Commercial Inkjet Head, MEMS '98.

8. Rasmussen et al, Utilization of Standard CMOS Layers for Microchannels, MEMS 1998, 1998 International Mechanical Engineering Congress and Exposition, Nov. 1998.

9. Smith J. H. et al, Material and Processing Issues for the Monolithic Integration of Microelectronics with Surface-Micromachined Polysilicon Sensors and Actuators, Micromachining and Microfabrication '95, SPIE, Oct. 1995.

10. Smith J. H. and S. T. Walsh, Selecting a Process Paradigm for an Emergent Disruptive Technology: Evidence from the Emerging Microsystems Technology Base, IEEE International Engineering Management Conference, Oct. 1998.

11. Tseng et al, A Microinjector Free of Satellite Drops and Ejected Droplets, MEMS 1998, 1998 International Mechanical Engineering Congress and Exposition, Nov. 1998.

12. White, F. M., Fluid Mechanics, McGraw-Hill, 1994.

13. Whitesides G., Unconventional Methods and Unconventional Materials for Microfabrication, June 1997, Transducers ' 97.

14. Wroblewski et al, MEMS Micro-valve Arrays for Fluidic Control, MEMS 1998, 1998 International Mechanical Engineering Congress and Exposition. 